\title{
Habilidades Sociais e Comorbidades Psiquiátricas de Mulheres Usuárias de Crack
}

\section{Social Skills and Psychiatric Comorbidities of Women Addicted to Crack}

\section{Habilidades sociales y comorbilidades psiquiátricas en mujeres usuarias de crack}

\section{Jéssica Limberger*}

Universidade do Vale do Rio dos Sinos - UNISINOS, São Leopoldo, Rio Grande do Sul, Brasil

\section{Ilana Andretta**}

Universidade do Vale do Rio dos Sinos - UNISINOS, São Leopoldo, Rio Grande do Sul, Brasil

\begin{abstract}
RESUMO
A literatura aponta que características clínicas como a depressão relacionamse com baixas habilidades sociais. Em mulheres usuárias de crack, tais questões ainda são pouco estudadas. Este estudo objetiva identificar as associações entre habilidades sociais e comorbidades psiquiátricas dessa população. Trata-se de um estudo transversal, quantitativo e correlacional, sendo utilizados como instrumentos: questionário de dados sociodemográficos e de uso de drogas, MINI (Mini International Neuropsychiatric Interview), Inventário de Habilidades Sociais (IHS-Del Prette), Screening Cognitivo do WAIS-III e SCID-II (Structured Clinical Interview for DSM Disorders). Participaram 62 mulheres em internação hospitalar, com média de idade de 33,45 anos $(D P=8,14)$. Mulheres com Episódio Depressivo Maior apresentaram escores significativamente menores no autocontrole da agressividade. Além disso, houve associação entre déficits em habilidades sociais e Transtorno de Personalidade Borderline. Evidencia-se a necessidade de considerar as comorbidades psiquiátricas como um dos fatores que prejudicam o uso das habilidades sociais. Intervenções como o Treinamento em Habilidades Sociais devem ser planejadas a partir de tais comorbidades.
\end{abstract}

Palavras-chave: mulheres, cocaína crack, habilidades sociais, transtornos relacionados ao uso de substâncias.

\begin{abstract}
The literature suggests that clinical characteristics such as depression are related to low social skills. In women users of crack, such issues are still little studied. Thus, the objective of this research is to identify associations between social skills and psychiatric comorbidities in this population. This is a transversal, quantitative and correlacional study, being used as instruments: data questionnaire sociodemographic and drug use, MINI (Mini
\end{abstract}


International Neuropsychiatric Interview), Social Skills Inventory (IHS-Del Prette), Cognitive Screening of the WAIS-III and SCID-II (Structured Clinical Interview for DSM Disorders). Participated 62 women in hospital, with a mean age of 33.45 years $(S D=8.14)$. Women with Major Depressive Episode had significantly lower scores in self-control aggression. In addition, there was an association between deficits in social skills and Borderline Personality Disorder. It's evident considering the psychiatric comorbidities as one of the factors that hinder the use of social skills. Interventions as the Social Skills Training should be planned from such comorbidities.

Keywords: women, crack cocaine, social skills, substance-related disorders.

\section{RESUMEN}

La literatura sugiere que las características clínicas, tales como la depresión están relacionadas con las habilidades sociales bajas. En mujeres usuarias de crack, estas cuestiones son todavía poco estudiadas. Por lo tanto, el objetivo es identificar asociaciones entre las habilidades sociales y las comorbilidades psiquiátricas en esta población. Se trata de un estudio transversal, cuantitativo y correlacional, siendo utilizados como instrumentos: cuestionario de datos sociodemográficos y el uso de drogas, MINI (Mini International Neuropsychiatric Interview), Inventario de Habilidades Sociales, examen cognitivo de la WAIS-III y SCID-II (Structured Clinical Interview for DSM Disorders). Participaron 62 mujeres en hospitalización, con una edad media de 33,45 años ( $D E=8,14$ ). Las mujeres con episodio depresivo mayor tenían puntuaciones significativamente más bajas en autocontrol de la agresividad. Además, hubo una asociación entre los déficits en habilidades sociales y trastorno de la personalidad borderline. La necesidad es considerar las comorbilidades psiquiátricas como uno de los factores que impiden el uso de las habilidades sociales. Intervenciones como el entrenamiento en habilidades sociales deben planificarse desde tales comorbilidades.

Palabras clave: mujeres, cocaína crack, habilidades sociales, trastornos relacionados con sustâncias.

\section{Introdução}

As habilidades sociais (HS) são apontadas pela literatura como importantes fatores de proteção no desenvolvimento, relacionando-se com o menor risco para o uso de drogas (Seitz, Wyrick, Orsini, Milroy \& Kenney, 2013; Torres, Arévalo, Cuevas, \& Rodríguez, 2006). Tais habilidades constituem um conjunto de comportamentos emitidos por uma pessoa em seu meio social, na expressão de seus sentimentos, desejos, atitudes, opiniões e direitos, de maneira adaptativa e assertiva, diminuindo a probabilidade de dificuldades futuras (Caballo, 2003).

Del Prette e Del Prette (2001) nomeiam cinco fatores de habilidades sociais. O enfrentamento com risco indica a capacidade de lidar com situações interpessoais, que necessitam a afirmação e defesa de direitos. A autoafirmação na expressão de afeto positivo compõe-se de habilidades como elogiar e agradecer elogios, defender em grupo outra pessoa e participar de conversa trivial. A conversação e 
desenvoltura social retrata a capacidade de lidar com situações com risco mínimo de reação indesejável, a partir de normas do relacionamento cotidiano. A autoexposição a desconhecidos e situações novas inclui a abordagem a pessoas desconhecidas, com maior risco de reação indesejável do outro. Por fim, o autocontrole da agressividade a situações aversivas, indica a expressão de desagrado ou raiva de maneira socialmente competente (Del Prette $\&$ Del Prette, 2001).

As habilidades sociais são desenvolvidas no decorrer do ciclo vital, sendo que as experiências de aprendizagem positivas são fatores que contribuem para o aumento do repertório de habilidades sociais (Del Prette, Ferreira, Dias, \& Del Prette, 2015). Diversos fatores dificultam a utilização das habilidades sociais, como a carência de modelos socialmente habilidosos, as experiências negativas de aprendizagem das habilidades sociais no decorrer do desenvolvimento e características clínicas como a depressão e a ansiedade (Fernandes, Falcone, \& Sardinha, 2012; Segrin, 2000).

Dentre as populações clínicas avaliadas, destacam-se as baixas habilidades sociais de usuários de drogas lícitas e ilícitas (Cunha, Carvalho, Kolling, Silva, \& Kristensen, 2007; Sintra, Lopes, \& Formiga, 2011; Sá \& Del Prette, 2011; Wagner \& Oliveira, 2015). Revisões sistemáticas sobre o tema apontam que a inabilidade social ocasiona piores respostas frente ao uso de drogas (Vieira \& Feldens, 2013; Wagner \& Oliveira, 2007). Especificamente em mulheres usuárias de crack, os relacionamentos interpessoais desempenham um importante papel na manutenção do uso de drogas, pois a rede de apoio acaba sendo substituída por relacionamentos entre usuários (Alves, Ribeiro, \& Castro, 2011; American Psychiatric Association, 2014; Limberger \& Andretta, 2015; Sayago, Lucena-Santos, Ribeiro, Yates, \& Oliveira, 2013).

Estudos sobre as habilidades sociais de mulheres usuárias de crack ainda são raros na literatura, conforme aponta uma recente revisão sistemática da literatura nacional e internacional sobre o uso de drogas e as habilidades sociais (Schneider, Limberger, \& Andretta, 2016). Além da avaliação das habilidades sociais nesta população, torna-se relevante a avaliação das comorbidades psiquiátricas, tendo em vista que baixas habilidades sociais relacionam-se com a depressão (Feitosa, 2013). Nessa perspectiva, conforme um estudo brasileiro, pessoas com depressão apresentam menor autocontrole da agressividade quando comparadas com pessoas não deprimidas (Fernandes, Falcone, \& Sardinha, 2012). Por sua vez, especialmente na população de usuários de crack, há maior prevalência de comorbidades psiquiátricas, que implicam em prejuízos como 0 aumento da agressividade, maior tempo de hospitalização e recaídas (Essau, 2008; Johnson, O'Leary, Striley, Abdallah, Bradford, \& Cottler, 2011; Mundt, Kastner, Larraín, Fritsch, \& Priebe, 2015). 
Considerando as baixas habilidades sociais de usuários de drogas apontados pela literatura, bem como os prejuízos decorrentes de comorbidades psiquiátricas, hipotetiza-se que baixas habilidades sociais estarão associadas à comorbidades psiquiátricas. Sendo assim, este estudo objetiva avaliar as habilidades sociais de mulheres usuárias de crack e identificar associações entre habilidades sociais e comorbidades psiquiátricas de tais mulheres.

\section{Método}

\subsection{Delineamento}

Trata-se de um estudo transversal, quantitativo e de alcance correlacional (Sampieri, Collado, \& Lucio, 2013).

\subsection{Participantes}

O tamanho da amostra foi definido através do software estatístico STATS 2.0, com nível desejado de confiança de $80 \%$. O cálculo foi realizado com base nos dados do Departamento de Informática do Sistema Único de Saúde (Datasus), considerando o número de mulheres em tratamento no SUS, no ano de 2013, devido ao uso de cocaína/crack, no estado do Rio Grande do Sul (Datasus, 2014). O resultado do cálculo apontou para uma amostra de 62 participantes.

Os critérios de inclusão foram: mulheres com idade entre 18 e 59 anos, abstinentes há pelo menos sete dias, com Transtorno por Uso de Substâncias, tipo crack, de acordo com os critérios diagnósticos do DSM-5 (APA, 2014), que estivessem entre o sétimo e o décimo quinto dia de internação hospitalar para desintoxicação devido ao uso do crack, via Sistema Único de Saúde (SUS). As participantes, em sua maioria solteiras $(62,9 \% ; n=39)$, possuíam média de 33,45 anos $(\mathrm{DP}=8,14)$. Grande parte da escolaridade referida foi 0 Ensino Fundamental Incompleto $(50,8 \% ; n=31)$ e Ensino Médio Incompleto $(23 \% ; n=14)$. De acordo com os Critérios Socioeconômicos Brasil, $32,3 \%(n=20)$ das mulheres pertencia à classe $C 1 ; 19,4 \%(n=12)$ à classe $C 2 ; 19,4 \%(n=12)$ à classe $E$ e $17,7 \%(n=11)$ à classe $D . O$ somatório das classes A e B foi de $11,3 \%(n=7)$.

Foram excluídas participantes com síndrome psicótica (verificada através do Mini International Neuropsychiatric Interview) e com prejuízos cognitivos (verificado através dos subtestes vocabulário e cubos do Screening Cognitivo do WAIS-III). Tais instrumentos serão descritos a seguir. 


\subsection{Instrumentos}

\section{Questionário de Dados Sociodemográficos e sobre o Uso de Drogas}

O questionário foi desenvolvido pelo grupo de pesquisa "Intervenções Cognitivo Comportamentais: Estudo e Pesquisa", sendo composto de questões fechadas sobre dados sociodemográficos, Critérios de Classificação Econômica Brasil, da Associação Brasileira de Empresas de Pesquisa (ABEP, 2015), dados familiares, padrão de uso de drogas (tipo e tempo de uso de cada droga utilizada), além de dados sobre o tratamento e critérios do DSM-5 (APA, 2014) para diagnóstico de Transtorno por Uso de Substâncias.

\section{Mini International Neuropsychiatric Interview (MINI)}

Trata-se de uma entrevista clínica padronizada breve, de livre acesso, compatível com os critérios diagnósticos do DSM-IV-TR (APA, 2002). Foi desenvolvida por Sheehan et al. (1998) e validada para o Brasil por Amorim (2000), com índices Kappa satisfatórios, demonstrando confiabilidade nas categorias diagnósticas $(0,86$ a 1$)$ e para Transtornos Psicóticos (0,62 a 0,95) (Amorim, 2000). A entrevista foi utilizada para avaliar a presença de síndrome psicótica (critério de exclusão) e comorbidades psiquiátricas (Episódio Depressivo Maior, Distimia, Episódio (Hipo) Maníaco, Transtorno de Pânico, Agorafobia, Fobia Social, Transtorno Obsessivo-Compulsivo, Transtorno de Estresse Pós-Traumático, Síndrome Psicótica, Anorexia Nervosa, Bulimia Nervosa e Transtorno de Ansiedade Generalizada), além do risco de suicídio.

\section{Screening Cognitivo do WAIS-III}

Trata-se de um teste de uso exclusivo dos psicólogos, desenvolvido por Wechsler (1997) e adaptado e padronizado para o Brasil por Nascimento (2004). O subteste vocabulário $(a=0,92)$ avalia a compreensão verbal a partir de palavras apresentadas ao examinando, que deve definir oralmente. O subteste cubos $(a=0,83)$ avalia a organização perceptual através de um conjunto de padrões geométricos bidimensionais que 0 examinando deve reproduzir usando cubos de duas cores. A correção deste instrumento foi realizada por dois juízes independentes, sendo acionado um terceiro juiz quando necessário para consenso. A partir da subtração do escore ponderado de vocabulário, para o escore ponderado de cubos, a diferença de três pontos ou mais indicou prejuízo cognitivo, conforme aponta Cunha (1993) e Feldens, Silva e Oliveira (2011). O prejuízo cognitivo configurou-se como critério de exclusão. 


\section{Inventário de Habilidades Sociais (IHS-Del Prette)}

Desenvolvido por Del Prette e Del Prette (2001) no contexto brasileiro, trata-se de um teste de uso exclusivo dos psicólogos que objetiva caracterizar as habilidades sociais em diferentes situações: trabalho, escola, família e cotidiano. O Inventário é de autorrelato, composto por 38 itens, em escala do tipo likert, com cinco pontos, que variam de nunca a raramente e sempre ou quase sempre. Possui Alfa de Cronbach de 0,75 e estabilidade teste-reteste $(r=0,90$; $p=0,001)$. A análise fatorial revelou uma estrutura de cinco fatores que reúnem habilidades sociais de: 1 ) enfrentamento/autoafirmação com risco; 2) autoafirmação de afeto positivo; 3 ) conversação e desenvoltura social; 4) autoexposição a desconhecidos e situações novas e 5) autocontrole da agressividade. Os resultados foram apurados de forma simplificada, com base na média simples dos valores obtidos, conforme sugere o manual. Valores situados no percentil 50 indicaram posição mediana, acima de $75 \%$ indicaram altos fatores em habilidades sociais e abaixo de $25 \%$ indicam déficits no repertório de habilidades sociais (Del Prette \& Del Prette, 2001).

\section{Structured Clinical Interview for DSM Disorders (SCID-II)}

Trata-se de uma entrevista semi-estruturada, utilizada em estudos observacionais e clínicos, que objetiva identificar diagnósticos de acordo com o DSM-IV-TR (APA, 2002). A partir desta entrevista, foram investigados os seguintes Transtornos de Personalidade: Histriônico, Narcisista; Borderline e Antissocial.

\subsection{Procedimentos}

Este estudo foi aprovado pelo Comitê de Ética em Pesquisa, sob parecer 012/2015. Os hospitais com internações via Sistema Único de Saúde foram escolhidos por conveniência, mediante contato explicitando os objetivos da pesquisa. Tais hospitais localizavam-se na região metropolitana e noroeste do estado do Rio Grande do Sul. Após Carta de Anuência dos hospitais, as participantes foram convidadas a participar da pesquisa. Foram explicados os objetivos da pesquisa e a voluntariedade no estudo, assegurando o sigilo dos dados e o anonimato. O Termo de Consentimento Livre e Esclarecido - TCLE foi lido em conjunto com a participante, e mediante a concordância em participar do estudo, foi assinado em duas vias, ficando uma via com a participante e outra via com a pesquisadora. A devolução dos resultados da pesquisa às participantes e aos respectivos hospitais foi ofertada.

Os dados foram coletados em um período de seis meses, pela pesquisadora e por seis integrantes do grupo de pesquisa, com treinamento específico para cada instrumento, conforme respectivas orientações de cada manual, além de supervisão semanal com a 
pesquisadora. Os instrumentos foram aplicados individualmente, no hospital, em local que permitisse a privacidade. A abstinência foi avaliada a partir do autorrelato, além de considerar o regime fechado de internação. Na maioria dos casos, os dados foram coletados em apenas um dia, e quando não houve tempo hábil, houve continuidade em outro dia.

Os dados foram analisados através do Statistical Package for Social Sciences - SPSS, versão 20.0. A análise descritiva contemplou frequências, porcentagem, média, mediana e desvio padrão da amostra, com estudo da distribuição de dados pelo teste de Kolmogorov-Smirnov. A fim de comparar as médias de habilidades sociais entre os diferentes grupos, utilizou-se o Teste $t$-Student nas variáveis com distribuição aproximadamente normal. Na comparação entre variáveis categóricas foi usado o teste Qui-quadrado de Pearson ou Exato de Fisher. Para critérios de decisão estatística adotou-se o nível de significância de $5 \%(p \leq 0,05)$.

\section{Resultados}

Foram entrevistadas 83 mulheres, sendo 21 excluídas por não contemplarem os critérios de inclusão, a saber: dez apresentavam síndrome psicótica, oito não completaram o preenchimento de todos os instrumentos e três não possuíam os critérios diagnósticos para Transtorno por Uso de Crack. Desta forma, 62 mulheres participaram da pesquisa.

A avaliação, a partir dos critérios diagnósticos do DSM-5 (APA, 2014) para Transtorno por Uso de Substâncias, demonstrou que uso de crack foi grave em $74,2 \% \quad(n=46)$ das mulheres, seguido de moderado $(21,0 \%, n=13)$ e leve $(4,8 \% ; n=3)$. No que diz respeito à avaliação geral das habilidades sociais, 37,1\% $(n=23)$ apresentou repertório acima da média no escore total, 25,8\% $(n=16)$ tiveram déficits, $21 \%(n=13)$ apresentaram repertório bastante elaborado e $16,1 \%(n=10)$ tiveram escore abaixo da média no referido escore. A maioria das mulheres apresentou déficits nos fatores conversação e desenvoltura social, autoexposição a desconhecidos e situações novas e autocontrole da agressividade, com resultados abaixo da média na autoafirmação e expressão de afeto positivo e resultados acima da média no enfrentamento e autoafirmação com risco, conforme detalhado na tabela 1. 
Tabela 1

Classificação dos Fatores de Habilidades Sociais a partir do IHS-Del Prette

\begin{tabular}{|c|c|c|c|c|c|c|c|c|c|c|}
\hline & \multicolumn{2}{|c|}{$\begin{array}{c}\text { Enfrentamento } \\
\text { com risco }\end{array}$} & \multicolumn{2}{|c|}{$\begin{array}{l}\text { Expressão de } \\
\text { afeto positivo }\end{array}$} & \multicolumn{2}{|c|}{$\begin{array}{c}\text { Conversação e } \\
\text { desenvoltura } \\
\text { Social }\end{array}$} & \multicolumn{2}{|c|}{$\begin{array}{c}\text { Autoexposição } \\
\text { a } \\
\text { desconhecidos }\end{array}$} & \multicolumn{2}{|c|}{$\begin{array}{c}\text { Autocontrole } \\
\text { da } \\
\text { Agressividade }\end{array}$} \\
\hline & $n$ & $\%$ & $n$ & $\%$ & $n$ & $\%$ & $n$ & $\%$ & $n$ & $\%$ \\
\hline Déficit & 8 & 12,9 & 15 & 24,2 & 25 & 40,3 & 22 & 35,5 & 24 & 38,7 \\
\hline $\begin{array}{l}\text { Abaixo da } \\
\text { média }\end{array}$ & 14 & 22,6 & 23 & 37,1 & 5 & 8,1 & 21 & 33,9 & 12 & 19,4 \\
\hline Médio & 2 & 3,2 & 0 & 0 & 0 & 0 & 0 & 0 & 7 & 11,3 \\
\hline $\begin{array}{l}\text { Acima da } \\
\text { média }\end{array}$ & 19 & 30,6 & 19 & 30,6 & 18 & 29,0 & 12 & 19,4 & 0 & 0 \\
\hline $\begin{array}{l}\text { Bastante } \\
\text { elaborado }\end{array}$ & 19 & 30,6 & 5 & 8,1 & 14 & 22,6 & 7 & 11,3 & 19 & 30,6 \\
\hline Total & 62 & 100 & 62 & 100 & 62 & 100 & 62 & 100 & 62 & 100 \\
\hline
\end{tabular}

Na avaliação clínica, o risco de suicídio foi apontado em $71 \%(n=44)$ dos casos. Foram identificadas comorbidades psiquiátricas através do MINI e transtornos de personalidade através da SCID-II, conforme tabela 2.

Tabela 2

Distribuição da Amostra quanto à Presença de Comorbidades Psiquiátricas e Transtornos de Personalidade

\begin{tabular}{lcc}
\hline Variável & $\mathrm{n}$ & $\%$ \\
\hline Episódio Depressivo Maior Atual & 22 & 35,5 \\
Episódio Depressivo Maior Passado & 13 & 21 \\
Transtorno de Ansiedade Generalizada & 20 & 32,3 \\
Episódio Maníaco Atual & 7 & 11,3 \\
Episódio Maníaco Passado & 5 & 8,1 \\
Transtorno de Pânico Vida Inteira & 6 & 9,7 \\
Transtorno de Pânico Atual & 2 & 3,2 \\
Distimia & 3 & 4,8 \\
Episódio Hipomaníaco Atual & 2 & 3,2 \\
Episódio Hipomaníaco Passado & 2 & 3,2 \\
Transtorno de Personalidade Antissocial & 13 & 21 \\
Transtorno de Personalidade Borderline & 12 & 19,4 \\
Transtorno de Personalidade Histriônico & 2 & 3,2 \\
\hline
\end{tabular}

A partir de tais comorbidades psiquiátricas, foram identificadas diferenças significativas em mulheres com maiores ou menores médias nas habilidades sociais, conforme podem ser observados na tabela a seguir. 
Tabela 3

Comparação entre Médias no Autocontrole da Agressividade e Comorbidades Psiquiátricas

\begin{tabular}{|c|c|c|c|c|c|c|c|}
\hline Variáveis & & & $n$ & Média & DP & $t$ & $P$ \\
\hline $\begin{array}{l}\text { Autocontrole da } \\
\text { agressividade }^{1}\end{array}$ & $\begin{array}{l}\text { Possui Episódio } \\
\text { Depressivo Maior }\end{array}$ & $\begin{array}{l}\text { Sim } \\
\text { Não }\end{array}$ & $\begin{array}{l}22 \\
40\end{array}$ & $\begin{array}{l}1,636 \\
2,208\end{array}$ & $\begin{array}{l}1,012 \\
0,832\end{array}$ & $\begin{array}{c}2,39 \\
5\end{array}$ & $0,020^{*}$ \\
\hline $\begin{array}{l}\text { Autocontrole da } \\
\text { agressividade }^{1}\end{array}$ & $\begin{array}{l}\text { Possui Transtorno de } \\
\text { Personalidade }\end{array}$ & $\underset{\text { Não }}{\text { Sim }}$ & $\begin{array}{l}25 \\
37\end{array}$ & $\begin{array}{l}1,653 \\
2,243\end{array}$ & $\begin{array}{l}1,006 \\
0,811\end{array}$ & $\begin{array}{c}2,54 \\
7\end{array}$ & $0,013^{*}$ \\
\hline
\end{tabular}

Nota, ${ }^{1}$ Variáveis com distribuição normal analisadas pelo Teste $t .{ }^{*} \mathrm{p}$ significativo ao nível de 0,05 .

A partir dos dados da tabela 3, identifica-se que mulheres com episódio depressivo maior tiveram médias significativamente menores no autocontrole da agressividade, além da presença de algum dos transtornos de personalidade avaliados (histriônico, narcisista, borderline e antissocial). Além disso, apresentar déficit no autocontrole da agressividade associou-se com ter transtorno de personalidade e ter transtorno de personalidade borderline, conforme pode ser observado na tabela 4.

Tabela 4

Associações entre déficits nas habilidades sociais e comorbidades psiquiátricas

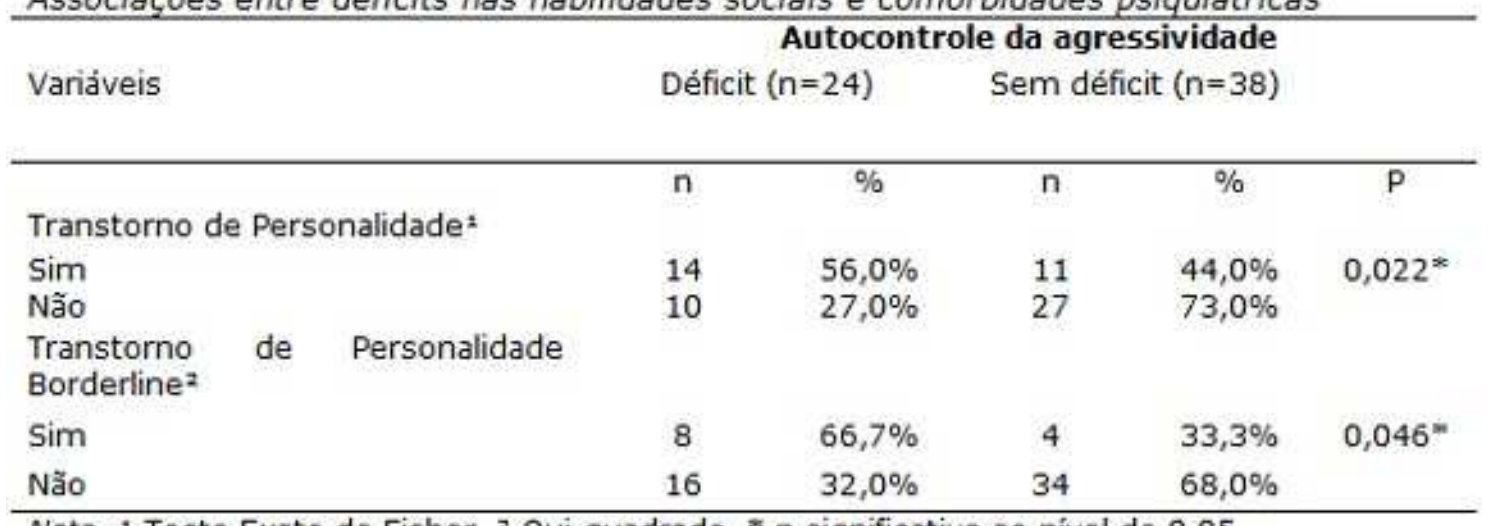

Nota. ${ }^{2}$ Teste Exato de Fisher. ${ }^{2}$ Qui-quadrado. ${ }^{*}$ p significativo ao nivel de 0,05.

\section{Discussão}

A partir do objetivo de avaliar as habilidades sociais de mulheres usuárias de crack, identificou-se que apenas no escore total e no enfrentamento/autoafirmação com risco foram encontrados resultados acima da média. Demais estudos brasileiros que compararam as HS de usuários e não usuários de drogas também não encontraram diferenças significativas no escore total de usuários de álcool, maconha, cocaína/crack (Wagner \& Oliveira, 2015; Sá \& Del Prette, 2011). Desta forma, o uso de drogas não necessariamente 
possui relação com o escore total de HS, mas sim com classes específicas (Sá \& Del Prette, 2011).

Destaca-se que os déficits encontrados na conversação e desenvoltura social foram os mais expressivos. Em um estudo brasileiro com 26 usuários de álcool (Cunha et al., 2007) e em um estudo português com 125 usuários de diferentes drogas ilícitas (Sintra et al., 2011), também foram encontrados déficits no referido fator. Considerando que as HS necessitam ser exercitadas para que o repertório seja aprimorado (Caballo, 2003), o isolamento social de usuários de crack pode dificultar ainda mais o uso de tais habilidades (Sayago, Lucena-Santos, Ribeiro, Yates, \& Oliveira, 2013). Nessa perspectiva, a carência de uma rede de apoio contribui em maiores prejuízos em mulheres usuárias de crack (Limberger \& Andretta, 2015), dificultando o exercício de habilidades de traquejo social no relacionamento cotidiano.

A partir da avaliação clínica, evidenciou-se comorbidades psiquiátricas, corroborando a literatura nacional e internacional (Hess, Almeida, \& Moraes, 2012; Mundt, Kastner, Larraín, Fritsch, \& Priebe, 2015). O Transtorno Depressivo Maior, presente neste estudo, condiz com outras evidências que indicam que tal transtorno associase ao uso de drogas, especialmente em mulheres (Essau, 2008). Em um estudo americano com mulheres usuárias de crack, o Episódio Depressivo Maior Atual foi um fator preditivo ao uso de crack (Johnson et al., 2011). Desta forma, a depressão também necessita ser considerada no tratamento do uso de crack em mulheres, tendo em vista que esta comorbidade psiquiátrica implica em sofrimento e prejuízos significativo no funcionamento social e demais áreas importantes da vida, além da perda de interesse, que pode impactar negativamente no tratamento do uso de drogas (APA, 2014).

Mulheres que possuíam Episódio Depressivo Maior Atual tiveram médias significativamente menores no autocontrole da agressividade, quando comparadas com mulheres sem Episódio Depressivo Maior Atual. De acordo com um estudo brasileiro, com 1.031 universitários, identificou-se uma correlação negativa entre depressão e o escore total de habilidades sociais, apontando que pessoas que possuem déficits nas HS percebem-se com um maior nível de sofrimento psicológico, ao serem comparadas com pessoas com maiores habilidades sociais (Feitosa, 2013). Em outro estudo brasileiro com 46 participantes, pacientes com depressão apresentaram médias significativamente menores no autocontrole da agressividade (Fernandes, Falcone, \& Sardinha, 2012). Nesse sentido, conforme Segrin (2010) baixas habilidades sociais podem tanto contribuir na ocorrência da depressão, como também serem sintomas de um quadro depressivo.

Neste estudo, apresentar um Transtorno de Personalidade associouse com déficits e menores médias no autocontrole da agressividade. 
Compreende-se que os transtornos de personalidade são acompanhados de sofrimento emocional, havendo prejuízos significativos na adaptação social e nas interações sociais (Feitosa, 2013). Dentre os transtornos de personalidade avaliados, 0 borderline associou-se significativamente com menores médias no autocontrole da agressividade. Tal habilidade social requer um automonitoramento e que possibilite condições no controle da raiva e na tolerância à frustração (Del Prette \& Del Prette, 2014; Vieira \& Feldens, 2013). Com isso, o exercício do autocontrole da agressividade é dificultado devido à impulsividade e o padrão de instabilidade nas relações interpessoais, característico do transtorno de personalidade borderline (APA, 2014; Wagner \& Oliveira, 2015).

Considerando que o fator de autocontrole da agressividade foi o único fator de habilidades sociais que se associou com as comorbidades psiquiátricas, percebe-se que há uma dificuldade das mulheres usuárias de crack em reagir a estímulos aversivos, onde a raiva é expressa de maneira desadaptativa, com maior probabilidade de problemas futuros. Tais questões podem ocorrer devido à escolha de recompensas imediatas e à impulsividade presente em usuários de crack, além da violência cotidiana dos contextos de uso (Alves, Ribeiro, \& Castro, 2011). Outro estudo brasileiro também ressalta esta questão, demonstrando a correlação negativa entre o uso de cocaína/crack e o autocontrole da agressividade (Sá \& Del Prette, 2011). Além disso, as dificuldades no autocontrole da agressividade podem acarretar em prejuízos nas demais habilidades sociais, pois há uma dificuldade na compreensão nas necessidades do interlocutor.

Diante de tais achados, compreende-se que o desenvolvimento de habilidades sociais específicas, como o autocontrole da agressividade, mostra-se relevante tanto no contexto do tratamento ao uso de drogas quanto nos demais contextos de interação. Sendo assim, há necessidade de intervenções, como o Treinamento em Habilidades Sociais para usuários de drogas, a fim de que um novo repertório seja desenvolvido, contribuindo para a qualidade das interações sociais (Schneider, Limberger, \& Andretta, 2016; Wagner \& Oliveira, 2007; Seitz, Wyrick, Orsini, Milroy, \& Kenney, 2013).

\section{Conclusão}

Este estudo possibilitou avaliar as habilidades sociais de mulheres usuárias de crack, sendo encontrados déficits específicos na conversação e desenvoltura social, auto-exposição a desconhecidos e situações novas e autocontrole da agressividade. Comorbidades psiquiátricas como o Episódio Depressivo Maior e o Transtorno de Personalidade Borderline associaram-se com baixo repertório e déficits no autocontrole da agressividade. Desta forma, os objetivos 
propostos foram contemplados, possibilitando novas compreensões sobre aspectos pouco explorados na literatura acerca das mulheres usuárias de crack.

A partir deste estudo, evidenciou-se que a avaliação das habilidades sociais de usuários de drogas necessita considerar as comorbidades psiquiátricas, pois estas podem estar dificultando o uso das habilidades sociais nos diferentes contextos de interação. Sugere-se que futuros estudos avaliem as habilidades sociais para além de instrumentos de autorrelato, utilizando protocolos de observação dos comportamentos emitidos. Além disso, sugere-se a avaliação dos demais Transtornos de Personalidade e a expansão do estudo para outras populações clínicas.

\section{Referências}

Alves, H. N. P., Ribeiro, M., \& Castro, C. D. (2011). Cocaína e Crack. In: Diehl, A., Cordeiro, D. C., \& Laranjeira, R. (Eds.), Dependência Química: prevenção, tratamento e políticas públicas (pp. 170-179). Porto Alegre: Artmed.

American Psychiatric Association [APA]. (2002). Manual diagnóstico e estatístico dos transtornos mentais. DSM IV-TR (4. ed.). Porto Alegre: Artes Médicas.

American Psychiatric Association [APA]. (2014). Manual Diagnóstico e Estatístico de Transtornos Mentais. DSM-5. Porto Alegre: Artmed.

Amorim, P. (2000). Mini International Neuropsychiatric Interview (MINI): validação de entrevista breve para diagnóstico de transtornos mentais. Revista Brasileira de Psiquiatria, 22(3), 106-115. doi: http://dx.doi.org/10.1590/S151644462000000300003

Associação Brasileira de Empresas de Pesquisa [ABEP]. (2015). Critérios de Classificação Econômica Brasil. Retrieved from http://www.abep.org/criterio-brasil

Caballo, V. E. (2003). Manual de avaliação e treinamento das habilidades sociais. São Paulo: Livraria Santos.

Cunha, J. A. (1993). Psicodiagnóstico-R. 4. ed. Porto Alegre: Artes Médicas.

Cunha, S. M., Carvalho, J. C. N., Kolling, N. M., Silva, C. R. \& Kristensen, C. H. (2007). Habilidades sociais em alcoolistas: um estudo exploratório. Revista Brasileira de Terapias Cognitivas, 3(1), 28-41. Retrieved from: http://pepsic.bvsalud.org/scielo.php?pid=S180856872007000100004\&script=sci_arttext 
Del Prette, Z. A. P., \& Del Prette, A. (2001). Inventário de Habilidades Sociais: Manual de aplicação, apuração e interpretação. São Paulo: Casa do Psicólogo.

Del Prette, A., \& Del Prette, Z. A. P. (2014). Psicologia das relações interpessoais: vivências para o trabalho em grupo. Petrópolis: Vozes.

Del Prette, Z. A. P; Ferreira, B. C., Dias, T. P., \& Del Prette, A. (2015). Habilidades sociais ao longo do desenvolvimento: perspectivas de intervenção em saúde mental. In: Murta, S. G., Leandro-Grança, C., Santos, K. B. (Eds.), Prevenção e Promoção em Saúde Mental: fundamentos, planejamento e estratégias de intervenção (pp.318-340). Novo Hamburgo: Sinopys.

Departamento de Informática do SUS [Datasus]. (2014). Retrieved from:

http://www2.datasus.gov.br/DATASUS/index.php?area $=0202$

Essau, C. A. (2008). Comorbidity of addictive problems: assessment and treatment implications. In: Essau, C. A. (Ed.), Adolescent Addiction: Epidemiology, Assessment and Treatment. London: Academic Press.

Feitosa, F. B. (2013). Habilidades Sociais e sofrimento psicológico. Arquivos Brasileiros de Psicologia, 65(1). 38-50.

Feldens, A. C. M., Silva, J. G. D., \& Oliveira, M. D. S. (2011). Avaliação das funções executivas em alcoolistas. Cadernos de Saúde Coletiva, 19, 164-171. Retrieved from http://www.cadernos.iesc.ufrj.br/cadernos/images/csc/2011_2/ artigos/csc_v19n2_164-171.pdf

Fernandes, C. S., Falcone, E. M. O., Sardinha, A. (2012). Deficiências em habilidades sociais na depressão: estudo comparativo. Psicologia: teoria e prática, 14(1). Retrieved from: http://pepsic.bvsalud.org/scielo.php?script=sci_arttext\&pid=S1 516-36872012000100014\&Ing=pt\&tIng=pt.

Hess, A. R. B., Almeida, R. M. M., \& Moraes, A. L. (2012). Comorbidades psiquiátricas em dependentes químicos em abstinência em ambiente protegido. Estudos de Psicologia, 17(1). 171-178. http://dx.doi.org/10.1590/S1413294X2012000100021

Johnson, J. E., O'Leary, C. C., Striley, C. W., Abdallah, A. B., Bradford, S., \& Cottler, L. B. (2011). Effects of Major Depression on Crack Use and Arrests among Women in Drug Court. Addiction, 106(7). 1279-1286. doi:10.1111/j.13600443.2011.03389.x.

Limberger, J., \& Andretta, I. (2015). Novas problemáticas sociais: o uso do crack em mulheres e a perspectiva de gênero. CS, 14, 41-65. doi: 10.18046/recs.i15.1965 
Mundt, A. P., Kastner, S., Larraín, S., Fritsch, R., \& Priebe, S. (2015). Prevalence of mental disorders at admission to the penal justice system in emerging countries: a study from Chile. Epidemiology and Psychiatric Sciences. 1-19. doi: $10.1017 /$ S2045796015000554

Nascimento, E. (2004). Adaptação e padronização brasileira da Escala de Inteligência Wechsler para Adultos. Porto Alegre: Casa do Psicólogo.

Sá, L. G. S., \& Del Prette, Z. A. P. (2011). Correlação entre habilidades sociais e nível de envolvimento com álcool, maconha e cocaína/crack em dependentes químicos. Anais do IV Seminário Internacional de Habilidades Sociais. Retrieved from:

http://www.fapeti.com.br/ocs/index.php/sihs/sihs2011/paper/vi ew/177

Sampieri, R. H., Collado, C. F., \& Lucio, M. P. B. (2013). Metodologia de Pesquisa. Porto Alegre: Penso.

Sayago, C. B., Lucena-Santos, P., Ribeiro, F., Yates, M. B., \& Oliveira, M. S. (2013). Fatores protetivos e de risco para o uso de crack e danos decorrentes de sua utilização: revisão de literatura. Aletheia, 42, 164-174. Retrieved from: http://pepsic.bvsalud.org/scielo.php?pid=S141303942013000300014\&script=sci_arttext

Schneider, J. A., Limberger, J., \& Andretta, I. (2016). Habilidades Sociais e Drogas: Revisão Sistemática da Produção Científica Nacional e Internacional. Avances en Psicología Latinoamericana. doi: 10.12804/apl34.2.2016.08

Segrin, C. (2000). Social skills deficits associated with depression. Clinical Psychology Review, 20(3). 379-403. http://dx.doi.org/10.1016/S0272-7358(98)00104-4

Seitz, C, M., Wyrick, D. L., Orsini, M. M., Milroy, J. J., \& Kenney, M. F. (2013). Coverage of Adolescent Substance Use Prevention in State Frameworks for Health Education: 10 Year Follow-Up. Journal of School Health, 83(1), 53-60. doi: 10.1111/j.17461561.2012.00747.x.

Sheehan, D. V., Lecrubier, Y., Sheehan, K. H., Amorim, P., Janavs, J., Weiller, E., Herqueta, T., Baker, R., \& Dunbar, G. C. (1998). The Mini-International Neuropsychiatric Interview (M.I.N.I.): the development and validation of a structured diagnostic psychiatric interview for DSM-IV and ICD-10. Journal Clinic Psychiatry, 59(supl. 20), 22-33.

Sintra, C. I. F., Lopes, P., \& Formiga, N. (2011). Condutas antissociais e delitivas e habilidades sociais em contexto forense. Psicol. Argum, 29, 383-399. Retrieved from: http://www2.pucpr.br/reol/index.php/PA?dd1=5296\&dd99=vie w 
Vieira, A. C. S., \& Feldens, A. C. M. (2013). Habilidades sociais, dependência química e abuso de drogas: uma revisão das publicações científicas dos últimos 6 anos. Trabalho de Conclusão de Curso em Pós-Graudação, Facultades Integradas de Taquara, Taquara, RS, Brasil. Retrieved from https://psicologia.faccat.br/blog/wpcontent/uploads/2013/07/Ana-Caroline-Sari-Vieira.pdf

Wagner, M. F., \& Oliveira, M. S. (2007). Habilidades sociais e abuso de drogas em adolescentes. Psicologia Clínica, 19(2). 101-116. http://dx.doi.org/10.1590/S0103-56652007000200008

Wagner, M. F., \& Oliveira, M. S. (2015). Habilidades sociais no abuso e na dependência de maconha. In: Del Prette, Z. A. P., Soares, A. B., Pereira-Guizo, C. S., Wagner, M. F., \& Leme, V. B. R. Habilidades sociais: diálogos e intercâmbios sobre pesquisa e prática. Novo Hamburgo: Sinopys Editora.

Wechsler, D. (1997). Weschsler Adult Intelligence Scale-III. San Antonio, TX: The Psychological Corporation.

\section{Endereço para correspondência \\ Jéssica Limberger}

Universidade do Vale do Rio dos Sinos - UNISINOS

Escola de Saúde, Programa de Pós-Graduação em Psicologia

Avenida Unisinos, 950, Cristo Rei, CEP 93020-000, São Leopoldo - RS, Brasil

Endereço eletrônico: jessica.limberger.psi@gmail.com

\section{Ilana Andretta**}

Universidade do Vale do Rio dos Sinos - UNISINOS

Escola de Saúde, Programa de Pós-Graduação em Psicologia

Avenida Unisinos, 950, Cristo Rei, CEP 93020-000, São Leopoldo - RS, Brasil

Endereço eletrônico: iandretta@unisinos.br

Recebido em: $17 / 02 / 2016$

Reformulado em: 29/09/2016

Aceito em: $21 / 10 / 2016$

\section{Notas}

* Psicóloga, Mestra em Psicologia Clínica. Doutoranda em Psicologia Clínica na Universidade do Vale do Rio dos Sinos. Bolsista CAPES/PROSUP.

** Psicóloga, Mestra e Doutora em Psicologia. Professora do Programa de PósGraduação em Psicologia da Universidade do Vale do Rio dos Sinos. 